\title{
Tyrosine kinase inhibitors: new molecules in non-small cell lung cancer (EGFR AND ALK)
}

\author{
Fernando Franco, Míriam Méndez*, Lourdes Gutierrez, Mariano Provencio \\ Puerta de Hierro Universitary Hospital, Madrid, Spain \\ *Corresponding Author: E-mail: mirimega@hotmail.com.
}

Received: October 25, 2016; Revised: February 22, 2017; Published: March 25, 2017

\begin{abstract}
Lung cancer is a tumor pathology that includes a group of neoplasms with different biological characteristics, which determine the course of the disease and the potential treatment response depending on the subtype diagnosed. Non-small lung carcinoma corresponds to $85 \%$ of all lung cancers. Adenocarcinoma is the most common histologic subtype of this group. In the United States approximately 130,000 new cases a year are diagnosed and in the world around a million. Adenocarcinoma is the most likely subtype, with possible molecular alterations (EGFR mutations and translocations in ALK), which are a therapeutic target. Knowledge of the molecular profile of the tumor and its biology has led us to identify therapeutic targets as protein tyrosine kinase, whose inhibition have demonstrated impact in terms of survival and quality of life for patients with these molecular alterations. However, the complex biology of non-small cell lung carcinoma gives the malignant cell the ability to generate mechanisms of resistance to these drugs and has forced us to extend the studies to control the disease by blocking these mechanisms.
\end{abstract}

\section{Keywords}

Lung cancer; EGFR inhibitors, ALK inhibitors

\section{Introduction}

Non-small lung carcinoma corresponds to $85 \%$ of lung cancers. Adenocarcinoma is the most common histologic subtype. In the United States approximately 130,000 new cases are reported per year [1]. Patients with advanced non-small cell lung cancer (NSCLC) usually receive systemic therapy. After histopathological diagnosis, an extensive molecular study is required to characterize the tumor. Regardless of the clinical characteristics of the patient, at least the mutational status of the epidermal growth factor receptor (EGFR) and ALK should be analyzed [2-4]. Activating mutations in EGFR tyrosine kinase are observed in approximately 15 percent of NSCLC adenocarcinoma in the United States and occur more frequently in nonsmokers [5]. In Asian populations, the incidence of these EGFR mutations is substantially higher, up to 62 percent [6].

In unselected NSCLC populations, the ALK rearrangement is a relatively rare event. The overall incidence of ALK gene rearrangements has been about 4 percent. The presence of ALK gene rearrangements in NSCLC tumors tends to occur independently of EGFR mutations. Similar frequencies of ALK gene rearrangements have been reported in Asian and Western populations. 


\section{EGFR inhibitors}

\section{First and second generation of EGFR inhibitors}

Patients with molecular alterations constitute a subpopulation with clinical characteristics, prognosis and response to different treatment (chemotherapy vs tyrosine kinase inhibitors) [5,7-9]. First-generation EGFR TKIs (including gefitinib and erlotinib) selectively target the receptor, through a competitive, reversible binding at the tyrosine kinase domain. However, despite good response there is evidence that after a variable period of time (5-13 months), the patients develop acquired resistance to EGFR-TKI therapy that negatively impacts their survival. Second generation TKIs (Afatinib, Dacomitinib) differ from first generation EGFR-TKIs because they form an irreversible link to the EGFR kinase domain and inhibit receptors as HER2 and HER4 and are active agents particularly in those with exon 19 deletion. The increasing knowledge in tumor biology and the mechanisms, by which resistance develops, have helped to develop new molecules that have already demonstrated a positive impact on response rates and survival [9-11].

\section{Third generation of EGFR inhibitors}

The T790M EGFR mutation is present in over $60 \%$ of cases of resistance to TKIs first and second generation. It is the resistance mechanism of EGFR most commonly known. This acquired resistance is mediated by a threonine-to-methionine mutation in EGFR at position 790 (EGFR T790M), activation of a bypass-signalling pathway such as mesenchymal-epithelial transition (MET) pathway, human epidermal growth factor 2 (HER2), or mitogen-activated protein kinase 1 (MAPK1) amplification, and histologic transformation to small-cell lung cancer or an epithelial-to-mesenchymal transition [12-15]. Following the acquisition of the mutation, the median survival is less than two years, so there is a need to develop new drugs that will control tumor growth in patients positive EGFR T790M.

\section{Osimertinib (AZD9291)}

AZD9291 approved by the FDA in November 2015, is a new molecule TKI third generation, which binds irreversibly to the residue kinase EGFR at the site of ATP binding by covalent bond formation, in patients who have acquired T790M mutation. After oral administration, it is metabolized AZD9291 that has two pharmacologically active forms AZ5104 and AZ7550. AZ7550 showed a comparable potency and selectivity profile similar to osimertinib, whereas AZ5104 in addition exhibited greater potency against exon 19 deletion and T790M mutants and wild-type EGFR. The first phase I dose escalation study was the AURA, made with EGFR mutated patients in progression with gefitinib or erlotinib treatment [11, 16-18]. The doses used were between 20 and $240 \mathrm{mg}$ per day. In the expansion phase, the analysis of the T790M mutation patients demonstrated an objective response rate of $67 \%$ (95\% Cl 52-70\%) compared to $21 \%$ in patients without the mutation. The rate of disease control in the overall analysis of all AURA studies was $91 \%$. The median progression-free survival was 9.6 months versus 3 months in patients without the mutation. The profile of side events is favourable, since they are few and mostly grades 1 and 2 . The most frequent were rash, diarrhea and nausea. Since no significant difference in response to higher doses was observed, $80 \mathrm{mg} /$ day is recommended until disease progression or unacceptable toxicity [16-18]. If it is necessary to reduce the dose, this should be done at $40 \mathrm{mg}$ per day, since tablets are $40 \mathrm{and} 80 \mathrm{mg}$. Its elimination is mainly hepatic and has not been studied in patients with renal insufficiency [16]. Current studies of liquid biopsy are presented as an option for detection of mutations in peripheral blood. This probably serves not only for diagnosis but also for monitoring; however, more studies are needed in this line. 


\section{$\underline{\text { Rociletinib (CO-16086) }}$}

Rociletinib, is a new oral selective covalent molecule EGFR inhibitor, with demonstrated activity against the T790M mutation, deletionn 19 and L858R [19-20]. Initial studies were performed in patients with acquired resistance to gefitinib and erlotinib. The subsequent inclusion of patients with mutation T790M has shown the particular activity of the drug in this subgroup of patients. Partial results of studies of a phase II study presented at ASCO 2015 reflect an objective response rate in this subgroup of $59 \%(95 \% \mathrm{Cl}$ 45-73) and rates of disease control $85 \%$. However, new combined analyses of the TIGER-X and TIGER-2 studies have shown that the actual response rate is only around $30 \%$. Among the most common adverse events are diarrhea and skin toxicity, most of them in grades 1 and 2 [21]. Cases of hyperglycemia and QT prolongation (>10\%) were frequently presented, in the latter case without reaching documented arrhythmias. Dose reductions occurred in $51 \%$ of patients, most commonly due to hyperglycemia (22\%) and QTC prolongation (11\%). Fifty-seven percent of patients had dose interruptions, which were most often due to hyperglycemia (22\%), QTc prolongation (10\%), and nausea (10\%). The average survival in patients was 8 months with the T790M mutation in patients where the 500-650 mg dose was administered. Although initially seeming a promising drug, the adverse event profile and response rates have prompted the FDA to give a negative opinion for accelerated approval of the drug [21-22].

$\underline{\mathrm{HM} 61713 \text { (BI 1482694) }}$

HM61713 is an irreversible kinase inhibitor that binds to a cysteine residue near the EGFR kinase domain. Preliminary studies in cell lines, has shown activity against T790M mutations and L858R. Studies are currently underway with Phase I / II with a dose of $800 \mathrm{mg} /$ day in patients with T790M mutation. Initial results show an overall response rate of $62 \%$ in this subgroup of patients. Disease control rate was $91 \%$ by independent assessment. The most common treatment-related adverse events included diarrhoea (55\%), nausea (37\%), rash (38\%) and pruritus (36\%), the majority were mild-to-moderate [23]. It seems a promising agent; however further studies to confirm therapeutic activity are needed. ELUXA 1 is an ongoing pivotal Phase II clinical trial, designed to further investigate the efficacy and safety of BI 1482694 in patients with NSCLC whose tumours acquired T790M-mediated resistance after first-line EGFR TKIs.

\section{$\underline{\text { ASP8273 }}$}

ASP8273 is another small molecule, irreversible EGFR TK inhibitor targeting EGFR mutations including T790M. In mouse xenograft models, ASP8273 achieved complete remission of tumors after 14 days of treatment. Phase I / II studies have established $400 \mathrm{mg} /$ day as the optimal dose. The most common adverse events were gastrointestinal toxicity and thrombocytopenia. Dose-limiting toxicities were reported at levels higher doses (400-600 mg). Maximum tolerated dose was established as $300 \mathrm{mg}$. The objective response rate in patients with T790M was $25 \%$ [24-25].

\section{$\underline{\text { PF-06747775 and EGF816 }}$}

They are other new small molecules that inhibit T790M; however, they are in early stages of study and results are expected to verify their antitumor activity in the clinic [26-30].

Similarly, there are clinical phase I-II trials to evaluate the effectiveness of combinations of third generation TKIs with various molecules, including MET and MEK inhibitors, as well as immunotherapy, among others. 


\section{ALK inhibitors}

Anaplastic lymphoma kinase (ALK) is a member of the insulin receptor tyrosine kinase family (RTK). The echinoderm microtubule-associated protein-like 4-anaplastic lymphoma kinase (EML4-ALK) fusion oncogene arises from an inversion on the short arm of chromosome 2 that joins EML4 to ALK. The resulting chimeric protein, EML4-ALK, contains an N-terminus derived from EML4 and a C-terminus containing the entire intracellular tyrosine kinase domain of ALK. ALK rearrangements occur in 3-7 \% of patients with NSCLC and are more common among patients with a non / light smoking history, adenocarcinoma histology, a younger age, female gender and in tumours wild type for EGFR and KRAS [31].

Anaplastic lymphoma kinase (ALK) gene rearrangements may be detected using fluorescence in situ hybridization (FISH) or immunohistochemistry (IHC). The gold standard assay for diagnosing is FISH. To be declared ALK positive, at least $15 \%$ of 50 evaluated tumor cells must harbour split signals. IHC may be a more practical approach to detecting ALK-positive NSCLC. Moreover, the absence of ALK expression in normal lung tissue makes IHC less cumbersome to interpret than FISH. In 2015, an IHC test using the D5F3 ALK antibody (Ventana) was approved after demonstration of concordance with the break apart FISH test, with $94 \%$ overall agreement [32].

\section{First generation of ALK inhibitors}

\section{Crizotinib (PF-02341066, Xalkori)}

Crizotinib is the first-generation ALK inhibitor approved for ALK-positive NSCLC (4). It also has activity against c-MET and ROS1 tyrosine kinases. It was approved in first line and in pretreated ALK positive NSCLC patients [33-34]. Response rates to crizotinib are approximately $60 \%$, which means there are a $40 \%$ of primary resistances. One reason is because some ALK translocations may not generate functional rearrangements in all patients [35]. There are secondary causes of resistance thought ALK kinase mutations and it may occur in $30 \%$ of the cases: L1196M, F1174L, and G1202R or C1156Y mutation [36] Activation of other oncogenes resulting in bypass signaling cascades such as EGFR, CKIT and KRAS or copy number gains of the ALK fusion gene [37] and pharmacokinetic failings of the drug itself due to inadequate systemic levels or poor CNS penetration and about $50 \%$ of patients develop CNS metastases during treatment with crizotinib [38-39] and in many cases, no definitive resistance mutation can be identified [40].

\section{Second generation of ALK inhibitors}

These drugs were developed to overcome crizotinib-resistant mutations and to improve activity in CNS disease (Table 1).

\section{Ceritinib}

Ceritinib is 20-fold more potent than crizotinib in terms of ALK selectivity [41]. It strongly inhibits resistance mutations, and especially L1196M, G1269A, I1171T and S1206Y mutations. However, ceritinib was not effective against G1202R and F1174C crizotinib-resistant mutations. The updated ASCEND-1 report on the evaluation of the efficacy of ceritinib at a daily dose of $750 \mathrm{mg}$ was recently presented. A total of 246 patients with ALK+ NSCLC were enrolled, including 163 pretreated with an ALK inhibitor (crizotinib or alectinib) and 83 ALK inhibitor naïve patients. The overall rate responses (ORRs) were $56.4 \%, 72.3 \%$ and $61.8 \%$ and the median of progression free survival (PFS) was 18.4, 9.0 and 6.9 months in the group pretreated with an ALK inhibitor, ALK inhibitor-naive patients and the overall population, respectively. 124 patients had measurable brain metastases at baseline: 28 patients pretreated with an ALK inhibitor and 8 
patients in the ALK inhibitor naive group. The intracranial ORRs were $36 \%$ (95 \% Cl 19-56 \%) in patients pretreated with an ALK inhibitor and $63 \%$ (95 \% Cl 25-92\%) in the ALK inhibitor-naive group [33,34]. The ASCEND-3 trial enrolled 124 patients, $40.3 \%$ with brain metastases, $46 \%$ of whom had no prior BRT (Brain Radiotherapy). The ORR was $63.7 \%$ (95 \% Cl54.6-72.2 \%). The intracranial ORR was $20 \%$ (95 \% Cl 2.5$55.6 \%$ [35]. Two randomized phase III trials are currently open to evaluate ceritinib versus first-line chemotherapy (ASCEND-4) and second-line chemotherapy (ASCEND-5).

\section{Alectinib}

Alectinib is a highly selective ALK inhibitor, in preclinical studies it showed activity against crizotinibresistant ALK mutations (L1196M, C1156Y and F1174L) and RET but not against InsR, IGF-R1 and ROS1. Japan was the first country to approve alectinib in patients with advanced ALK-rearranged NSCLC, based on the results of the phase I/II AF-001JP trial targeting patients with ALK+ NSCLC who had not previously been treated with crizotinib or other ALK inhibitors [42,43]. In the phase II trial, 46 patients were treated and 43 of them (93.5 \%) achieved an ORR (95\% Cl 82.1-98.6). The 2-year PFS rate was $76 \%$ (95\% Cl 60-86 \%) and the 2-year OS was $79 \%$ (95 \% Cl 63-89\%). A phase I/II trial with 47 ALK+ NSCLC patients who progressed on or were intolerant to crizotinib. The ORR was $55 \%$ and the subset analysis of 21 patients with CNS metastases at baseline showed a disease control rate approximating $90 \%$. The recommended dose for phase II studies was $600 \mathrm{mg}$ BID [44-46].

\section{$\underline{\text { Brigatinib }}$}

Brigatinib is a novel potent oral drug that has demonstrated ability to overcome crizotinib resistance mutations, including G1202R and activity against ROS1 and inhibits mutant EGFR, including T790M [47-49]. Brigatinib showed antitumor activity in patients treated with crizotinib with an objective response rate of $71 \%$ and in crizotinib-naive patients of $100 \%$. The median PFS was 13.4 months in the subgroup with prior crizotinib therapy [50]. A pivotal, randomized, phase 2 trial of brigatinib in patients with crizotinib-resistant ALK+ NSCLC has completed accrual.

\section{$\underline{\text { Entrectinib }}$}

Entrectinib is a novel, orally available, selective tyrosine kinase inhibitor of the Trk family of tyrosine kinases, ROS1 and ALK. Rearrangements in NTRK1 were recently described in approximately $3 \%$ of NSCLC that were negative for other oncogenic driver mutations [51]. Entrectinib has demonstrated in vitro and in vivo antitumour activity against various TRK, ROS1 or ALK-driven xenograft models of different human cancers (NPM-ALK-driven lymphoma and EML4-ALK-driven NSCLC) Entrectinib has adequate oral bioavailability and can efficiently cross the blood-brain barrier in mice with intracranially injected $\mathrm{NClH} 2228$ EML4-ALK cells [52]. Entrectinib $400 \mathrm{mg} / \mathrm{m} 2$ is the dose recommended for the trials phase II [53].

\section{Third generation of ALK inhibitors}

Lorlatinib (PF-06463922)

Lorlatinib has been identified as an orally novel reversible available ATP-competitive selective targeting of a specific residue leucine at position 1198 (L1198) detected in only approximately $25 \%$ of kinases, brain penetrant inhibitor of ALK (including all known mutations) and the c-Ros oncogene 1 (ROS1). In vitro and preclinical studies it is $\sim 10$-fold more potent against wildtype EML4-ALK and $\sim 40$-fold more potent against EML4-ALK L1196M compared with crizotinib [54]. Importantly, lorlatinib has demonstrated activity in patients whose tumors harbor the highly resistant mutation ALK G1202R. This mutation confers resistance 
to other next-generation ALK inhibitors.

\section{Other drugs for crizotinib resistant tumor}

\section{$\underline{\text { ASP3026 }}$}

This is an ALK inhibitor active against crizotinib-resistant ALK-positive advanced tumors [55].

\section{$\underline{X-376}$ and X-396 (Xcovery)}

These are more potent inhibitors of ALK but less potent inhibitors of MET compared to crizotinib. Moreover, X-396 could potently inhibit ALK with two point mutations (L1196M and C1156Y) associated with acquired resistance to crizotinib and there is preclinical data about CNS penetration [56].

\section{CEP-28122}

CEP-29122 is a potent and selective ALK inhibitor which showed selectivity in preclinical studies against ALK compared to various types of tyrosine kinases, including InsR, IGF-R1 and c-MET. CEP- 37440 is an ALK inhibitor and focal adhesion kinase (FAK) [57-58].

\section{$\underline{\text { HSP90 inhibitors }}$}

Another possibility to overcome resistance to ALK inhibitors includes the combination with HSP90 inhibitors such as retaspimycin hydrochloride (IPI-504) and ganetespib (STA-9090) that have demonstrated clinical activity in ALK+ NSCLC patients [60-62]. Several clinical trials are evaluating HSP90 inhibitors in combination with ALK inhibitors. Crizotinib is combined with ganetespib in crizotinib-naive patients and in crizotinib-resistant disease. In other studies crizotinib is combined with onalespib (AT13387), while ceritinib is combined with AUY922.

Table 1. Main molecular characteristics of second and third-generation ALK inhibitors.

\begin{tabular}{|c|c|c|c|c|}
\hline Drug & $\begin{array}{c}\text { Targets other } \\
\text { than ALK }\end{array}$ & $\begin{array}{l}\mathrm{L} 1196 \mathrm{M} \\
\text { resistance } \\
\text { mutation }\end{array}$ & $\begin{array}{c}\text { C1156Y } \\
\text { resistance } \\
\text { mutation }\end{array}$ & $\begin{array}{c}\text { G1202R } \\
\text { resistance } \\
\text { mutation }\end{array}$ \\
\hline Ceritinib & $\begin{array}{c}\text { IGF-R1, InsR, } \\
\text { ROS1 }\end{array}$ & Sens. & Res. & Res. \\
\hline Alectinib & LTK, GAK & Sens. & Sens. & Res. \\
\hline Brigatinib & ROS1, EGFR & Sens. & Sens. & Sens. \\
\hline Entrectinib & $\begin{array}{l}\text { TrkA, TrkB, } \\
\text { TrkC, ROS1 }\end{array}$ & Sens. & Sens. & ND \\
\hline Lorlatinib & ROS1 & Sens. & ND & Sens. \\
\hline TSR-011 & $\begin{array}{c}\text { TrkA, TrkB, } \\
\text { TrkC }\end{array}$ & Sens. & ND & ND \\
\hline ASP3026 & ROS1, ACK & Sens. & ND & ND \\
\hline$x-396$ & MET & Sens. & Sens. & ND \\
\hline
\end{tabular}

\section{Conclusions}

Current knowledge of the biology of NSCLC, allows the development of new molecules that are able to control the disease and have an impact on disease control, survival and quality of life. The new thirdgeneration EGFR TKIs demonstrate efficacy in patients with EGFR resistance mutations patients while the new ALK inhibitors show activity in patients with ALK rearrangements and mutations. These benefits alone have already allowed the registration of some of them. Results from other studies are pending, as well as those related to combinations with other drugs. 


\section{References}

[1] R. Siegel, J. Ma, Z. Zou, A. Jemal. CA Cancer J. Clin. 64(1) (2014) 9-29.

[2] N. Lindeman, P. Cagle, M.B. Beasley et al. Arch. Pathol. Lab. Med. 137(6) (2013) 828-860.

[3] T.J. Lynch, D.W. Bell, R. Sordella, et al. N. Engl. J. Med. 350(21) (2004) 2129-2139.

[4] E.L. Kwak, Y.J. Bang, D.R. Camidge, et al. N. Engl. J. Med. 363(18) (2010) 1693-1703.

[5] R. Rosell, E. Carcereny, R. Gervais, et al. Lancet Oncol. 13(3) (2012) 239-246.

[6] F.-Y. Niu, Y.-L.Wu. Exp. Hematol. Oncol. 3(1) (2014) 2.

[7] N.B. Leighl, N. Rekhtman, W.A. Biermann, et al. J. Clin. Oncol. 32(32) (2014) 3673.

[8] A. Chi, S. Remick, W. Tse. Biomark. Res. 1(1) (2013) 2.

[9] C.K. Lee, Y.-L. Wu, P.N. Ding, et al. J. Clin. Oncol. 33(17) (2015) 1958-1965.

[10] A.O. Walter, R.T. Sjin, H.J. Haringsma, et al. Cancer Discov. 3(12) (2013) 1404-1415.

[11] D.A. Cross, S.E. Ashton, S. Ghiorghiu, et al. Cancer Discov. 4(9) (2014) 1046-1061.

[12] S. Kobayashi, T.J. Boggon, T. Dayaram, et al. N. Engl. J. Med. 352 (2005) 786-792.

[13] J.A. Engelman, K. Zejnullahu, T. Mitsudomi, et al. Science 316 (2007) 1039-1043.

[14] K. Takezawa, V. Pirazzoli, M.E. Arcila, et al. Cancer Discov. 2 (2012) 922-933.

[15] T.M. Kim, A. Song, D.-W. Kim, et al. J. Thorac. Oncol. 10 (2015) 1736-1744.

[16] P.A. Jänne, J.C. Yang, D.W. Kim, et al. N. Engl. J. Med. 372(18) (2015) 1689.

[17] S.S. Ramalingam, J. Yang, C. Lee, et al. J. Thoracic. Oncol. 10(9_suppl 2) (2015) S319.

[18] C.A. Eberlein, D. Stetson, A.A. Markovets, et al. Cancer Res. 75(12) (2015) 2489-500.

[19] A.O. Walter, R.T. Sjin, H.J. Haringsma, et al. Cancer Discov. 3 (2013) 1404-1415.

[20] L.V. Sequist, J.C. Soria, J.W. Goldman, et al. N. Engl. J. Med. 372(18) (2015) 1700-1709.

[21] J.C. Soria, D.R. Camidge. N. Engl. J. Med. 374 (2016) 2296-2297

[22] FDA Briefing Document Oncologic Drugs Advisory Committee Meeting. NDA 208542 Rociletinib. April 12,2016

[23] K.-O. Lee, M.Y. Cha, M. Kim, et al. Cancer Res. 74(19) (2014) LB-100.

[24] H. Sakagami, S. Konagai, H. Yamamoto, et al. Cancer Res. 74(19 Supplement) (2014) 1728.

[25] Y. Jia, J. Juarez, M. Manuia, G. Lelais, et al. Cancer Res. 74(19 Supplement) (2014) 1734.

[26] S. Kasibhatla, J. Li, C. Tompkins, et al. Cancer Res. 74(19 Supplement) (2014) 1733.

[27] Y. Goto, H. Nokihara, H. Murakami, et al. ASCO Meet Abstr. 33(15_suppl) (2015) 8014.

[28] G. Lelais, R. Epple, P.-Y. Michellys, et al. Cancer Res. 75(15 Supplement) (2015) 2585.

[29] Study For Patients with NSCLC EGFR Mutations (Del 19 or L858R +/- T790M). ClinicalTrials.gov Identifier: NCT02349633.

[30] A. Ullrich, J. Schlessinger. Cell 61(2) (1990) 203-212.

[31] D. Camidge, S. Kono, A. Flacco, et al. Clin. Cancer Res. 16(22) (2010) 5581-5590.

[32] J.J. Cui, M. Tran-Dubé, H. Shen, et al. J. Med. Chem. 54(18) (2011) 6342-6363.

[33] A.T. Shaw, S.H. Ou, Y.J. Bang, et al. N. Engl. J. Med. 371(21) (2014) 1963-1971.

[34] B.J. Solomon, T. Mok, D.W. Kim, et al. N. Engl. J. Med. 371(23) (2014) 2167-2177.

[35] E. Felip, S. Orlov, K. Park, et al. J. Clin. Oncol. 33 (2015) 8060.

[36] J.M. Heuckmann, H. Balke-Want, F. Malchers, et al. Clin. Cancer Res. 18(17) (2012) 4682-4690.

[37] Y.L. Choi, M. Soda, Y. Yamashita, et al. N. Engl. J. Med. 363(18) (2010) 1734-1739.

[38] R. Katayama, A.T. Shaw, T.M. Khan et al. Sci. Transl. Med. 4(120) (2012) 120 ra17.

[39] J. Gainor, S.H. Ou, J. Logan, L.F. Borges, A.T. Sahw. J. Thorac. Oncol. 8(12) (2013) 1570-1573.

[40] D. Costa, A.T. Shaw, S.H. Ou, et al. J. Clin. Oncol. 33(17) (2015) 1881-88.

[41] R. Doebele, A. Pilling, D. Aisner, et al. Clin. Cancer Res. 18(5) (2012) 1472-1482. 
[42] L. Friboulet, N. Li, R. Katayama, et al. Cancer Discov. 4(6) (2014) 662-673.

[43] K. Kinoshita, K. Asoh, N. Furuchi, et al Bioorg. Med. Chem. 20(3) (2012) 1271-1280.

[44] T. Seto, K. Kiura, M. Nishio, et al. Lancet Oncol. 14(7) (2013) 590-598.

[45] T. Tamura, T. Seto, K. Nakagawa, et al. Radiat. Oncol. 90 (2014) S6.

[46] S. Gadgeel, L. Gandhi, G. Riely, et al. Lancet Oncol. 15 (2014) 1119-1128.

[47] J. Wu, J. Savooji, D. Liu. J. Hematol. Oncol. 9 (2016) 9.

[48] R. Squillace, R. Anjum, D. Miller, et al. Cancer Res. 73(8 Suppl) (2013) Abstract \#5655.

[49] V. Rivera, F. Wang, R. Anjum, et al. Cancer Res. 72(8 Suppl) (2012) Abstract \#1794.

[50] D. Camidge, L. Bazhenova, R. Salgia, et al. J. Clin. Oncol. 33 (2015) 8062.

[51] A. Vaishnavi, M. Capelletti, A.T. Le, et al. Nat. Med. 19(11) (2013) 1469-1472.

[52] E. Ardini, M. Menichincheri, C. De Ponti, et al. Mol. Cancer Ther. 8(12 Suppl) (2009) A243.

[53] M. Patel, T. Bauer, S. Liu, et al. J. Clin. Oncol. 33 (2015) 2596.

[54] T. Johnson, P. Richardson, S. Bailey, et al. J. Med. Chem. 57(11) (2014) 4720-4744.

[55] T. Li, P. LoRusso, M. Maitland, et al. J. Hematol. Oncol. 9 (2016) 23.

[56] C. Lovly, J. Heuckmann, E. de Stanchina, et al. Cancer Res. 71(14) (2011) 4920-4931.

[57] M. Cheng, M. Quail, D. Girgrich, et al. Mol. Cancer Ther. 11 (2012) 670-679.

[58] I. Sullivan, D. Planchard. Ther. Adv. Med. Oncol. 8(1) (2016) 32-47.

[59] L. Sequist, S. Gettinger, N. Sanzer, et al. J. Clin. Oncol. 28(33) (2010) 4953-4960.

[60] E. Normant, G. Paez, K. West, et al. Oncogene 30(22) (2011) 2581-2586.

[61] M. Socinski, J. Goldman, I. El-Harary, et al. Clin. Cancer Res. 19(11) (2013) 3068-3077. 